\title{
RESEARCHES ON THE CHEMOTHERAPY OF PROTOZOAL INFECTIONS
}

\author{
By NobUTARo ISHII \\ The National Institute of Health of Japan, Tokyo
}

\section{CONTENTS}

\author{
I. Chemotherapy of Malaria \\ II. Chemotherapy of Spirochetosis \\ III. Chemotherapy of Trypanosomiasis
}

IV. Chemotherapy of Leishmaniasis

V. Chemotherapy of Entamebiasis

VI. Chemotherapy of ${ }^{\circ}$ Trichomoniasis

Researches on the chemotherapy of protozoal infections have been carried out for several years in our laboratory for the purpose of discovering new methods of treating these discases.

\section{Chemotherapy of Malaria}

The therapeutic activity of numerous drugs on canary malaria has been determined by Rohr's method for plasmoquine test. With a view to pursuing the investigations, we sought the chemical collaborations of Dr. E. Ochiai and Dr. K. Tsuda of the Pharmaceutical Laboratory of University of Tokyo.

The effective drugs on canary malaria (Plasmodium relictum) were applied for human cases in order to confirm their activity against malaria.

\section{(A) Sulfonamide Compounds}

A total of 53 sulfonamide compounds were tested in this laboratory. Sulfadibromobenzene and sulfadichlorobenzene seemed to be appliable for human malaria as compared with the activity of quinine on canary malaria. Accordingly sulfadibromobenzene were applied for three tertian cases with desirable results. (Table 1,2 )

Table 1. Sulfonamide

Sulfanilamide

3,5-Dibromo-sulfanilamide

Sulfaguanidin

p-Aminobenzolsulfonacetamide

Diseptal A

4 (4'-Amino-3', $5^{\prime}$-dibromobenzene-sulfonamide)-benzene-sulfon-dimethylamide

Diseptal B

Diseptal C

$\mathrm{p}$-( $\mathrm{p}^{\prime}$-Aminophenyl)-benzene-sulfonamide

Sulfanilanilide

1-Sulfanilamido-3,5-dibromobenzene

1-Sulfanilamido-3,5-dichlorobenzene

Sulfapyridine

2-(4'-Amino- $3^{\prime}, 5^{\prime}$-dibromobenzene-sulfonamido)-pyridine

2-Sulfanilamido-6-methylpyridine

3-Sulfanilamido-2, 6-lutidine

2-Sulfanilamido-5-chloropyridine

2-Sulfanilamido-5-bromopyridine 
2-Sulfanilamido-5-aminopyridine

2-(4'-Amino-3', 5'-dibromobenzene-sulfonamido)-5-aminopyridine

Sulfathiazole

Sulfamethylthiazole

2-(4'-Amino-3', 5'-dibromobenzene-sulfonamido)-4-methyl-5-bromothiazole

2-(4'-Amino-3', 5'-dibromobenzene-sulfonamido)-5-bromothiazole

2-Sulfạilamido-4-mețyl-5-carbothoxythiazole

4-Sulfanilamido-1-phenyl-2, 3-dimethyl-5-pyrazolone

2-Sulfanilamido-chinoline

3-Sulfanilamido-chinoline

2-Sulfanilamido-benzothiazole

2-Sulfanilamido-5-methoxy-benzothiazole

$\mathrm{N}^{4}$-Dimethylsulfanilamide

Marfanilamide

Prontosil rubrum

3-Carboxy-4-aminoazobenzene-4' ${ }^{\prime}$-sulfonamide

Rubiazol

Proseptasine

$2-\left(N^{4}-\right.$ Sulfanilylsulfanilamido $)-4-$ methylthiazole

$\mathrm{N}^{\prime}$-Dimethyl-N"-( $\mathrm{N}^{\prime 4}$ sulfanilylsulfanil $)$-sulfanilamide

$\mathrm{N}^{\prime}$-Dimethyl- $\mathrm{N}^{4}-\left(\mathrm{N}^{\prime 4}-\left(\mathrm{N}^{\prime / 4}-\right.\right.$ sulfanilyl-sulfanilyl $)$-sulfanilyl $)$-sulfanilamide

Phonocasil

$2-\left(\mathrm{N}^{4}\left(\mathrm{~N}^{\prime 4}\right.\right.$-Sulfanilyl-sulfanilyl)-sulfanilamido)-4-methyl-thiazole

2-Sulfanilamide-6-methoxy-benzothiazole

2-Sulfanilamido-6-ethoxy-benzothiazole

2-Sulfanilamido-5-bromo-benzothiazole

2-Sulfanilamido-6-chloro-benzothiazole

7-(4'-Sulfonamidophenylazo)-6-hydroxy-benzothiazole

7-(4'-Sulfonamidophenylazo)-2-methyl-6-hydroxy-benzothiazole

2-Sulfanilamido-pyrimidine

2-Sulfanilamido-4-methylpyrimidine

2-Sulfanilamido-4, 6-dimethylpyrimidine

1-Sulfanilamido-5-bromo-naphthalene

1-Sulfanilamido-4-bromo-naphthalene

1-Sulfanilamido-5, 8-dibromo-naphthalene

\section{Table 2}

\begin{tabular}{|c|c|c|c|}
\hline Quinine hydrochloride & $\begin{array}{l}\text { M.E.D. } \\
1: 800\end{array}$ & $\begin{array}{l}\text { M.T.D. } \\
1: 200\end{array}$ & $\begin{array}{c}\text { M.T.D. : M.E.D. } \\
1: 4\end{array}$ \\
\hline Sulfadibromobenzene & $1: 200$ & 1: 50 & $1: 4$ \\
\hline Sulfadichlorobenzene & $1: 400$ & $1: 200$ & $1: 2$ \\
\hline
\end{tabular}

\section{(B) Benzothiazole and benzothiazolone (2) compounds}

Sixteen benzothiazole and three benzothiazolone compounds were tested with negative results on canary malaria. (Table 3 )

Table 3. Benzothiazole, benzothiazolone

2-Sulfanilamido-benzothiazole

2-Sulfanilamido-5-methoxy-benzothiazole

2-Sulfanilamido-6-methoxy-benzothiazole

Sulfanilamido-6-ethoxy-benzothiazole

Sulfanilamido-5-bromo-benzothiazole 
2-Sulfanilamido-6-chloro-benzothiazole

7-(4'-Sulfonamidophenylazo)-6-hydroxy-benzothiazole

7-(4'-Sulfonamidophenylazo)-2-methyl-6-hydroxy-benzothiazole

2-( $\beta$-Diethylaminoethylamino)-benzothiazole

$2-(\gamma$-Diethylaminopropylamino)-benzothiazole

2-( $($-Diethylaminopropyl-acetylamino $)$-benzothiazole

2 - $(\gamma$-Diethylamino- $\beta$-oxy-propylamino $)$-benzothiazole

$2-(\gamma$-Diethylaminopropylamino)-5-methoxy-benzothiazole

$2-(\gamma$-Diethylaminopropylamino $)-6-$ methoxy-benzothiazole

2-Amino-3 ( $\gamma$-diethylaminopropyl)-benzothiazole

2-Amino-3 ( $\gamma$-diethylaminopropyl)-6-chloro-benzothiazole

3-( $\beta$-Diethylaminoethyl)-benzothiazolone (2)

3 -( $\beta$-Diethylaminopropyl)-benzothiazolone (2)

$3-(\gamma-$ Diethylaminopropyl $)-6-$ chloro-benzothiazolone $(2)$

\section{(C) Quinazolone compounds}

Nine quinazolone compounds were tested with negative results on canary malaria. (Table 4)

Table 4. Quinazolone (4)

6-Amino-quinazolone (4)

3-Methyl-6-amino-quinazolone (4)

3-Methyl-8-amino-quinazolone (4)

3-Methyl-6-chloro-8-Amino-quinazolone (4)

8-( $\beta$-Diethylaminoethylamino)-quinazolone (4)

3-Methyl-6-( $\beta$-diethylaminoethyl-amino)-quinazolone (4)

3-Methyl-7-( $\beta$-diethylaminoethyl-amino)-quinazolone (4)

3-Methyl-8-( $\beta$-diethylaminoethyl-amino)-quinazolone (4).

3-Methyl-6-chloro-8-( $\beta$-diethylaminoethyl-amino)-quinazolone (4)

\section{(D) Quinazoline compounds}

Fourteen quinazoline compounds were examined with some expectations in their activities on canary malaria. (Table 5)

Table 5. Quinazoline

4-( $\beta$-Diethylaminoethylamino)-quinazoline

4-( $\beta$-Diethylaminoethylamino)-8-nitro-quinazoline

4-( $\beta$-Diethylaminoethylamino)-6,8-dichloro-quinazoline

4-( $\gamma$-Diethylamin $>-\alpha$-methylpropylamino)-6,8-dichloro-quinazoline

4-( $\beta$-Diethylaminoethylamino)-6,8-dibromo-quinazoline ,

4-( $\gamma$-Diethylamino- $\alpha$-methyl-propylamino)-6,8-dibromo-quinazoline

4-Methoxy -6-( $\beta$-diethylaminoethylamino)-quinazoline

4-Methoxy-8-( $\beta$-diethylaminoethylamino)-quinazoline

4-Methoxy-7-( $\beta$-diethylaminoethylamino)-quinazoline

4-Methoxy-7-( $\gamma$-diethylaminopropylamino)-quinazoline

$4-(\gamma$-Diethylamino- $\alpha$-methylpropyl-amino)-6-methoxy-quinazoline

$2-(\gamma$-Diethylamino-propylamino)-quinazoline

4-Sulfanilamido-quinazoline

4-Sulfanilamido-6; 8-dibromo-quinazoline

\section{(E) Benzotriazole compounds}

Five benzotriazole compounds were tested with negative results on canary malaria. (Table 6) 


\section{Table 6. Benzotriazole}

5-Methoxybenzotriazine

1-( $\beta$-Diethylaminoethyl)-6-methoxy-benzotriazole

1 -( $\gamma$-Diethylamino- $\alpha$-methyl-propyl)-6-methoxy-benzotriazole

$1-(\delta$-Diethylamino- $\alpha$-methyl-butyl)-6-methoxy-benzotriazole

1-Methyl-3-( $\delta$-diethylamino- $\alpha$-methyl-butylamino)-4-amino-benzole

\section{(F) Benzoquinoline compounds}

Seven benzoquinoline compounds were tested with negative results on canary malaria (Table 7)

Table 7. Benzoquinoline

\section{7, 8-Benzoquinoline}

2-Methyl-5-methoxy-6-acetylamino-7,8-benzoquinoline

2-Methyl-4-diethylaminoethylamino-5 6-benzoquinoline

2-Methyl-4-diethylaminoethylamino-7, 8-benzoquinoline

2-Methyl-4-diethylaminoethylamino-6-methoxy-7:8-bénzoquinoline

2-Methyl-4-diethylaminoisopentylamino-7,8-benzoquinoline

2-Methyl-4-diethylaminoisopentylamino-6-bromo-7,8-benzoquinoline

\section{(G) Diphenylsulfone and approximate compounds}

Sixteen compounds were tested with the results of undesirable action on canary malaria. Promin, as Coggeshall (1911) published, has a slight activity on canary malaria. (Table 8)

Table 8. Diphenylsulfone and approximate compounds

4, 4'-Diamino-diphenylsulfone

4, 4'-Diamino-3', 5'-dibromo-diphenylsulfone

4-Amino-3', 5'-dibromo-diphenylsulfone

4-Amino-4'-nitro-diphenylsulfone

4, 4'-Dinitro-diphenylsulfone

4-Nitro-diphenylsulfone

4-Nitro-3'; 5'-dibromo-diphenylsulfone

4-Nitro-4'-amino-3', 5'-dibromo-diphenylsulfone

4, 4'-Diamidino-diphenylsulfone

4, 4'-Diamidino-diphenylsulfoxyde

4, 4'-Diamidino-stilbene

4, 4'-Diguanidino-diphenylsulfone

4, 4'-Diguanidino-diphenylsulfoxyde

4, 4'-Diguanidino-diphenylsulfide

Promin

4-Aminophenyl-3'-aminopyridyl-(6 $\left.6^{\prime}\right)$-sulfone-N, $\mathrm{N}^{\prime}$-didextrose sulfonate

\section{(H) Quinine derivatives}

Forty two quinine derivatives, collaborated by Dr. E. Ochiai; were examined and it was found that certain derivatives exhibited a malaricidal action on canary.

(Table 9,10 )

Q 4 (6-Amino-dihydro-cinchonidin) was applied in five human tertian cases with satisfactory results. 
Table 9. Quinine deriva ives

Q 1

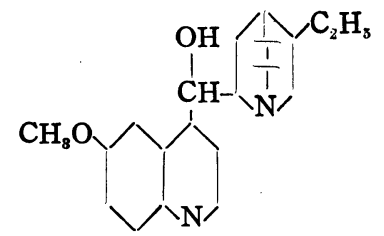

Q 2

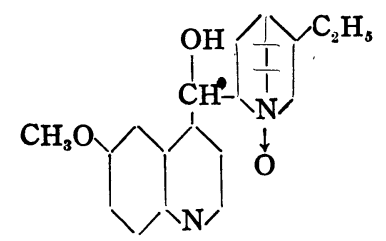

Q 48

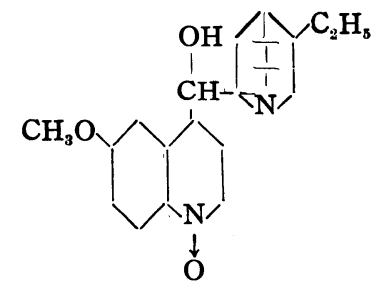

Q 36

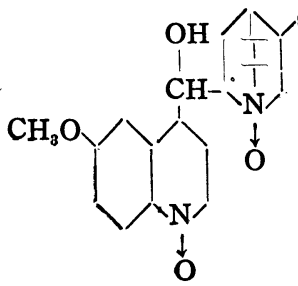

Q 4<smiles>CCC12CN3CC(CCCC3C1CCCN)C2CN</smiles>

Q 15

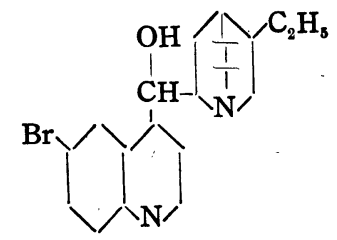

Q 41

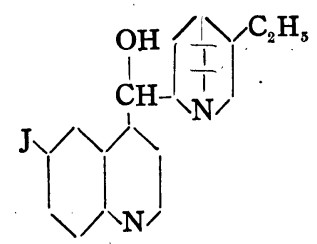

Q 43

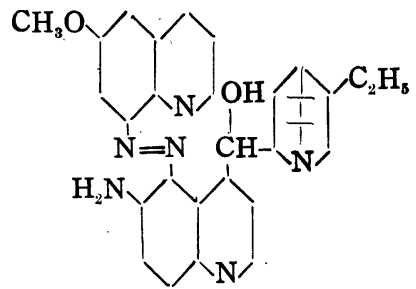

Q 37

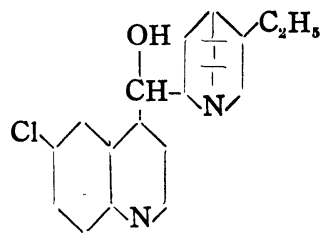

Q 16

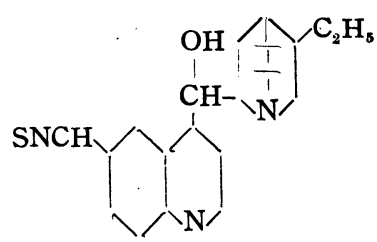

Q 47

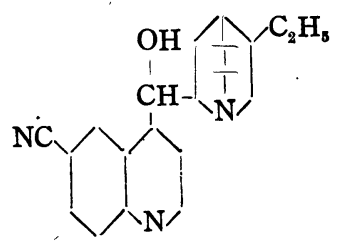

Q 6

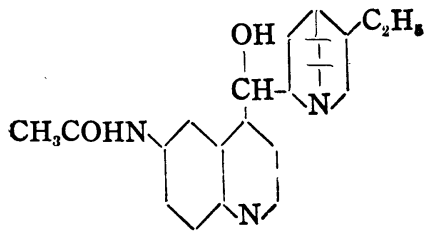

Q 21

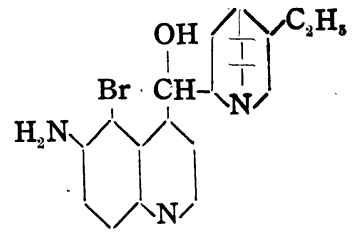

Q 13

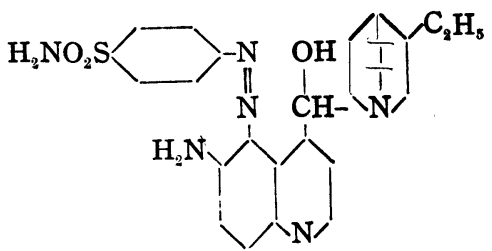




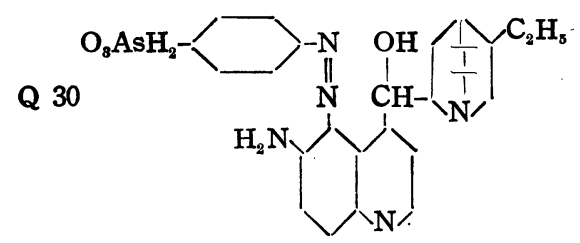

Q 11

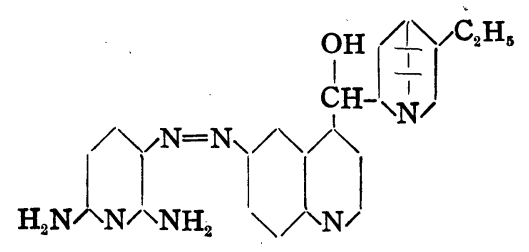

Q 40

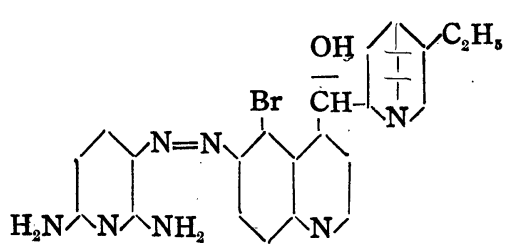

Q 33

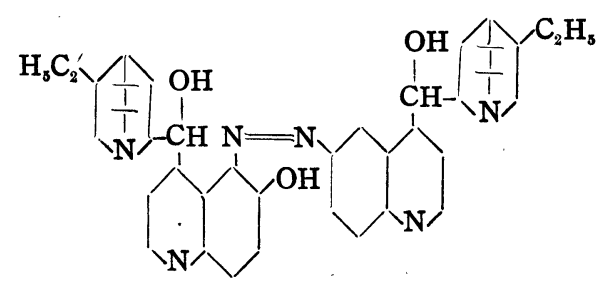

Q 38

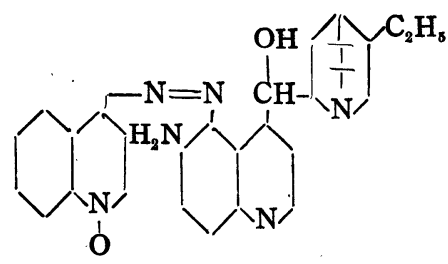

Q 42

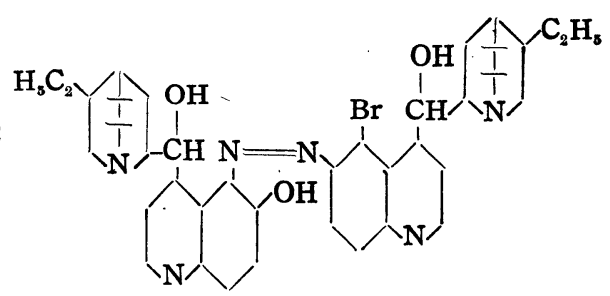

Q 7

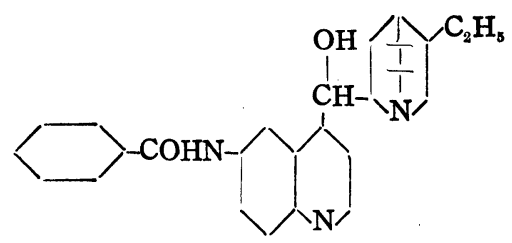

Q 46

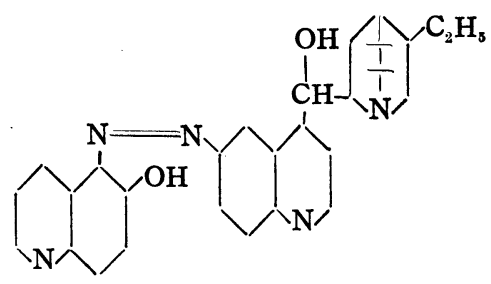

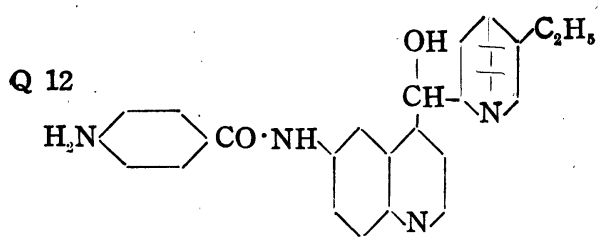

Q 18

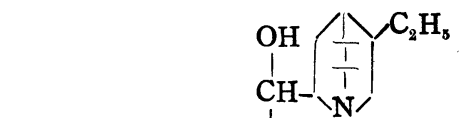<smiles>O=S(=O)(O)OC1CCCCC1C=NNC1CCC2N=CCC(C(O)O)C2C1</smiles>

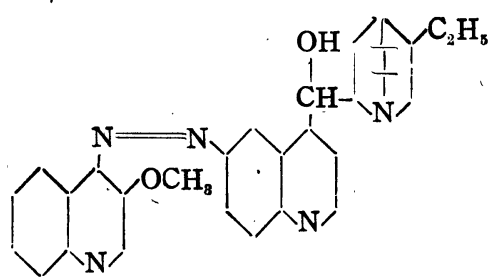

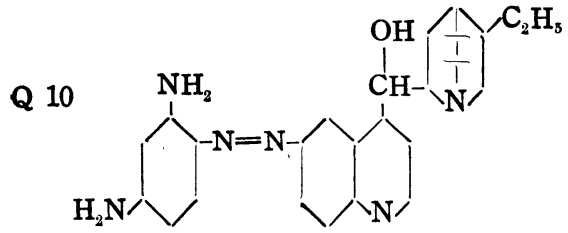

Q 49

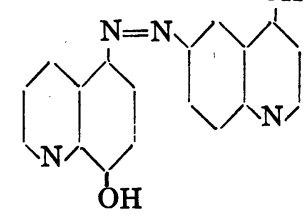

Q 23

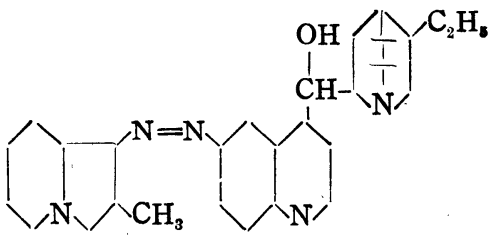


Q 19

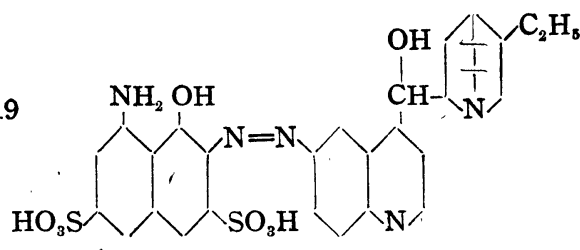

Q 17

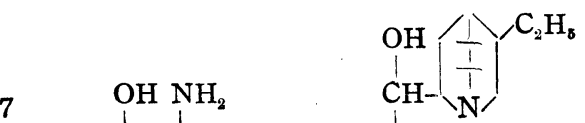

Q 27

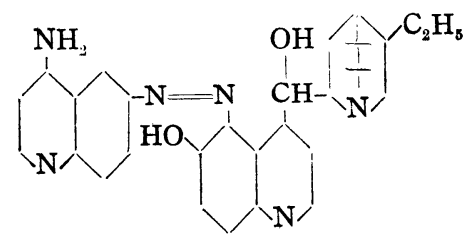

Q 39

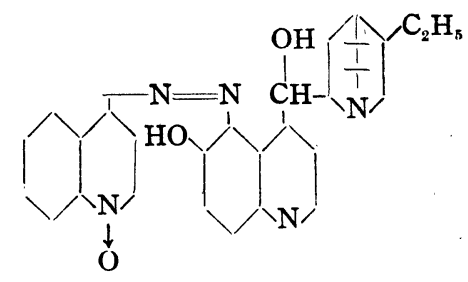

$\mathrm{H}_{8} \mathrm{C}_{2} \backslash \bigwedge$

Q 14<smiles>CCC1CCCCC1C(CC)N=CC(C)C</smiles>

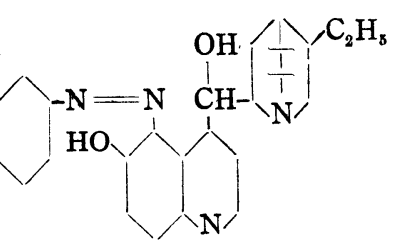

Q 22

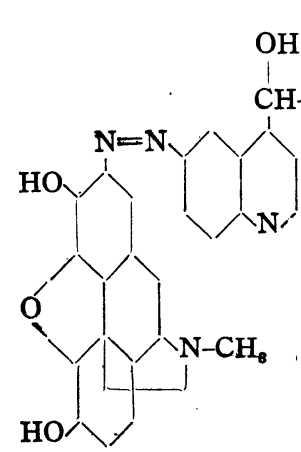

Q 28

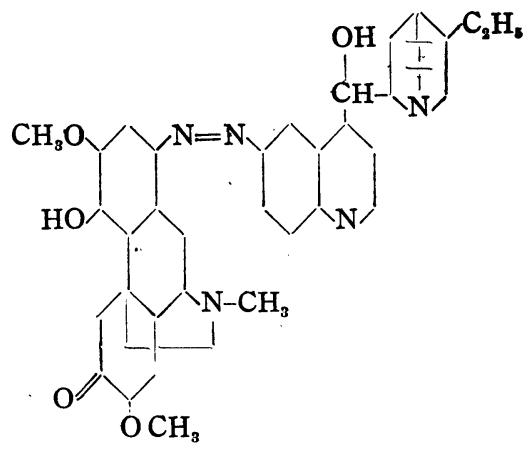

Q 31

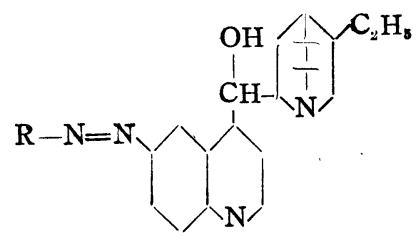

Q 5

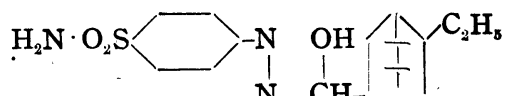

(n) $\overbrace{\mathrm{N}}$

Q 20

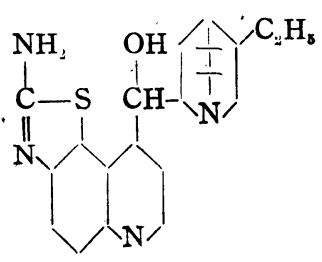

Q 29<smiles>CCCC1CN2CCC1C(C(O)C1CCCN=C3CCC(N)CC31)C2</smiles>

Q 32

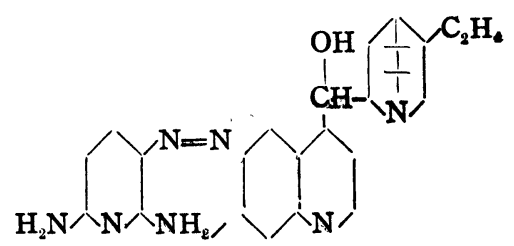




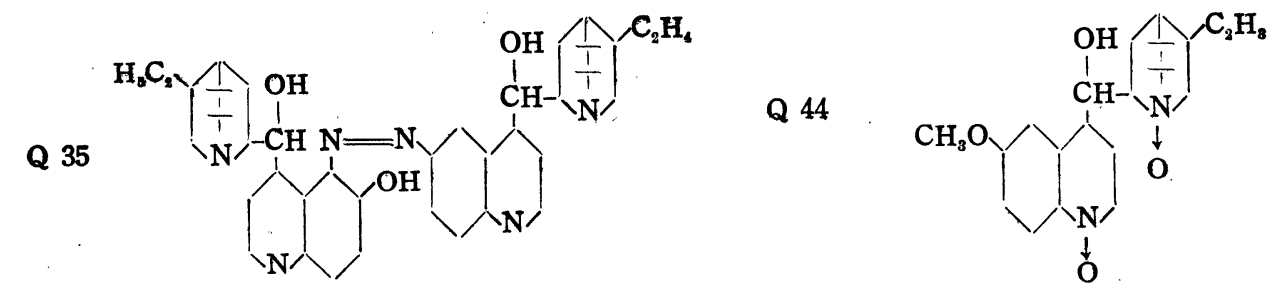

Table 10.

\begin{tabular}{l|l|l|c}
\hline \hline & M.E.D. & M.T.D. & M.T.D.: M.E.D. \\
\hline Quinine hydrochloride & $1: 800$ & $1: 200$ & $1: 4$ \\
Atebrin & $1: 3000$ & $1: 100$ & $1: 30$ \\
Plasmoquine & $1: 50000$ & $1: 1500$ & $1: 30$ \\
Sulfa-dibromobenzene & $1: 200$ & $1: 50$ & $1: 4$ \\
Sulfadichlorobenzene & $1: 400$ & $1: 200$ & $1: 2$ \\
Q 4 & $1: 800$ & $1: 50$ & $1: 16$ \\
Q 15 & $1: 1600$ & $1: 100$ & $1: 16$ \\
Q 41 & $1: 1600$ & $1: 200$ & $1: 8$ \\
Q 37 & $1: 1600$ & $1: 400$ & $1: 4$ \\
Q 46 & $1: 1600$ & $1: 100$ & $1: 16$ \\
Q 14 & $1: 800$ & $1: 100$ & $1: 8$ \\
Q 11 & $1: 800$ & $1: 100$ & $1: 8$ \\
Q 23 & $1: 800$ & $1: 50$ & $1: 16$ \\
Atebrin (Asano) & $1: 3000$ & $1: 50$ & $1: 60$ \\
No.'19 (Taguchi) & $1: 800$ & $1: 100$ & $1: 8$ \\
\hline
\end{tabular}

M.E.D. . . . . . Minimum effective dose M.T.D. . . . . . Maximum tolerated dose

\section{(I) Miscellaneous compounds}

1) Modified atebrin was tested, in collabiration with Dr. M. Asano, which exhibited the same malaricidal power on canary malaria as atebrin. The drug is simpler in its manufacture than atebrin. The malaricidal action in five tertian cases was demonstrated with satisfactory results. (Table 11)

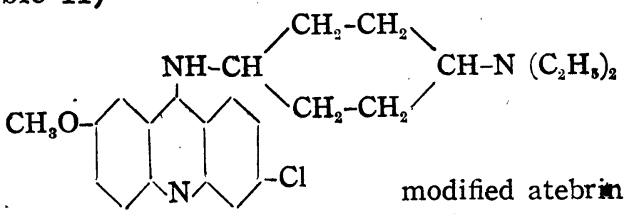

2) Chemical compound of quininbisulfate and sulfadibromobenzene (produced by Dr. Taguchi) was examined in canary malaria with effective results. Consequently we applied this drug in five tertian cases, which showed satisfactory results. (Table 11)

Table 11

\begin{tabular}{|c|c|c|c|c|}
\hline Drug & M.E.D. & M.T.D & M.T & M.E.D. \\
\hline 1) Asano & $\begin{array}{l}1: 3.000 \\
1: 10000\end{array}$ & $\begin{array}{l}1: 50 \\
1: 500\end{array}$ & $\begin{array}{l}1: 60 \\
1: 20\end{array}$ & $\begin{array}{l}\text { oral } \\
\text { subcutan }\end{array}$ \\
\hline 2) Taguchi & 1: 8000 & $1: 100$ & $1: 8$ & oral \\
\hline
\end{tabular}

M.E.D. . . . . . . . Minimum effective dose

M.T.D. . . . . . Maximum tolerated dose 


\section{(J) New provocative drugs and complete cure of malaria}

Cepharanthin, a drug designed for tuberculosis, was found to act as a provocative for extra-erythrocytic plasmodia in our experimental investigations on canary malaria.

Isotetrandrin and hypo-epistephanin have also provocative actions; however, the action of these two drugs is not quite the same as that of cepharanthin. The erythrocytic plasmodia appear mostly within 1-2 hours after cepharanthin injections, while the appearance of erythrocytic plasmodia is within about 20 hours after isotetrandrin and hypoepistephanin application.

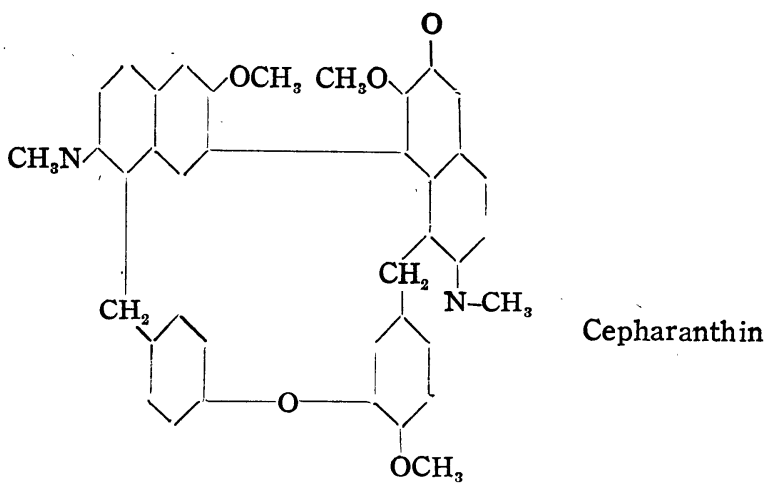

The recently produced evidence that cepharanthin stimulates the endothelial cells . explains the fact that the extra-erythrocytic forms are provoked out from the cells into the blood stream through endothelial stimulation.

\section{Complete cure of malaria}

It has been known that complete cure of malaria is difficult by means of quinine, atebrin, plasmoquine, etc., the reason being these drugs are not able to destroy the extraerythrocytic forms of plasmodium. We, therefore, endeavored to bring about complete cure by cepharanthin in combination with malaricidal drugs, and our observations were fairly satisfactory. This means that the malaricidal drugs act on the erythrocytic forms after they have been changed from extra-erythrocytic forms by means of cepharanthin.

In the first experiment, cepharanthin was used together with quinine hydrochloride, in 5 to 7 successions every other day or 7 times every day. Five days after the therapy . nos completed, cepharanthin was applied as a provocative. According to this experiment desirable results were not obtained, but the provocative action of cepharanthin was ascertained.

In the second experiment, we observed the behavior of erythrocytic plasmodium after either cepharanthin or hypoepistephanin was applied combined with quinine in a succession of 5 days. The plasmodium appeared wthin the erythrocytes from the 3rd to 5th day after the drugs were given. According to this observation, 5-day treatment followed by a 3-day interval seems to be better; therefore, for a complete cure this mode of treatment should be repeated several times. Accordingly 3 courses of treatment were applied in this experiment.' As shown in table 12, cepharanthin, isotetrandrin and 
hypoepistephanin were used together with quinine-hydrochloride. Ten days after these courses of treatment were carried out, cepharanthin was used as a provocative; 2 cases $(66 \%)$ out of 3 showed complete cure of chronic malaria. (Table 12)

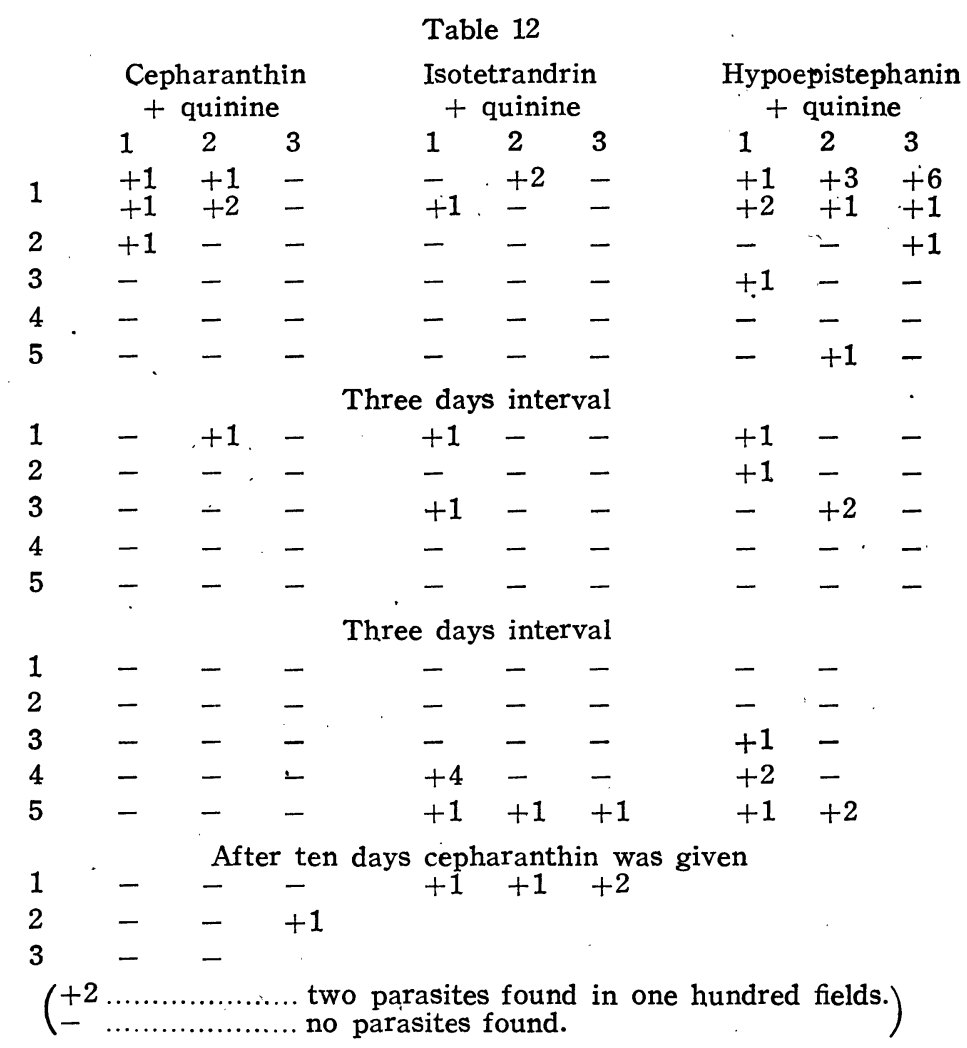

In the third experiment, atebrin and plasmoquine were used together with cepharanthin. Three cases $(60 \%)$ out of 5 , which were treated with cepharanthin and with atebrin or plasmoquine showed complete cure; however, 3 cases in which cepharanthin was not applied but atebrin alone was used, showed incomplete cure by provocative cepharanthin. (Table 13)

Table 13

\begin{tabular}{|c|c|c|c|c|c|c|c|c|}
\hline & \multicolumn{3}{|c|}{ Atebrin } & \multicolumn{5}{|c|}{$\begin{aligned} & \text { Atebrin } \\
+ & \text { cepharanthin }\end{aligned}$} \\
\hline & 1 & 2 & 3 & 1 & 2 & 3 & 4 & 5 \\
\hline 1 & $\bar{z}$ & $\begin{array}{l}+2 \\
+4\end{array}$ & $\overline{+8}$ & \pm & $\overline{-}$ & \pm 2 & $\begin{array}{l}+1 \\
+1\end{array}$ & \pm \\
\hline 2 & - & +4 & +6 & - & - & - & - & - \\
\hline 3 & - & +2 & - & - & - & - & - & - \\
\hline 4 & - & +2 & - & - & - & - & - & - \\
\hline 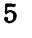 & - & - & - & - & - & - & - & - \\
\hline \multicolumn{9}{|c|}{ Three days interval } \\
\hline 1 & - & - & - & - & - & - & - & - \\
\hline 2 & - & - & - & - & - & - & - & - \\
\hline 3 & - & - & - & - & - & - & - & 一 \\
\hline 4 & - & - & - & - & - & - & - & - \\
\hline 5 & - & - & - & - & - & - & - & - \\
\hline
\end{tabular}


Three days interval

\begin{tabular}{|c|c|c|c|c|c|c|c|c|}
\hline $\mathbf{1}$ & - & - & - & - & - & - & - & - \\
\hline & - & - & - & - & - & - & - & 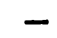 \\
\hline & - & - & - & - & - & - & - & - \\
\hline & \multicolumn{8}{|c|}{ After seven days cepharanthin was given } \\
\hline & +1 & +1 & +1 & - & - & - & - & - \\
\hline & +1 & +1 & +1 & - & - & - & +1 & - \\
\hline & +1 & +1 & +1 & +1 & - & - & +1 & \\
\hline
\end{tabular}

Two (60\%) out of 3 cases, which were tried with cepharanthin and plasmoquine, showed complete cure; however, 3 cases treated only with plasmoquine showed incomplete cure. (Table 14)

Table 14

\begin{tabular}{|c|c|c|c|c|c|c|}
\hline & \multicolumn{3}{|c|}{ Plasmoquine } & \multicolumn{3}{|c|}{$\begin{aligned} & \text { Plasmoquine } \\
+ & \text { cepharanthin }\end{aligned}$} \\
\hline & 1 & 2 & 3 & 1 & 2 & 3 \\
\hline 1 & \pm 1 & $\underline{ \pm}$ & \pm & $\underline{+1}$ & \pm & $\begin{array}{r}+1 \\
+4\end{array}$ \\
\hline 2 & - & - & - & - & - & +9 \\
\hline 3 & - & - & - & - & - & - \\
\hline 4 & - & - & - & - & - & - \\
\hline \multicolumn{7}{|c|}{ Five days interval } \\
\hline 1 & +3 & - & - & - & - & - \\
\hline 2 & - & - & - & - & - & - \\
\hline 3 & - & - & - & - & - & - \\
\hline 4 & +2 & - & - & - & - & - \\
\hline 5 & - & - & - & - & - & - \\
\hline \multicolumn{7}{|c|}{ Five days interval } \\
\hline 1 & - & - & - & - & - & - \\
\hline 2 & - & - & - & - & - & - \\
\hline 3 & - & - & - & - & - & - \\
\hline 4 & - & - & - & - & - & - \\
\hline 5 & - & - & - & - & - & - \\
\hline \multicolumn{7}{|c|}{ After seven days cepharanthin was given } \\
\hline 1 & +1 & - & +1 & - & - & +1 \\
\hline 2 & - & - & & - & - & \\
\hline 3 & & +1 & & - & - & \\
\hline
\end{tabular}

Conclusion: From the above experiments it is concluded that the complete cure of malaria is expected when cepharanthin together with malaricidal drugs are employed.

\section{Chemotherapy of Spirochaetosis.}

The efficacy of a number of drugs ágainst spirochaetosis recurrentis (duttoni) in white mice was tested in our laboratory. We found that sulfapyridine, sulfathiazole and sulfamethylthiazole showed some activity on spirochaetes.

\section{(1) Sulfonamide compounds.}

Forty three sulfonamide compounds were examined in experimental spirochaetosis recurrentis of mice. (Table 15,16 ) 
Sulfanilamide

Table 15

Sulfanilguanidine

p-Aminobenzolsulfonacetamidè

Diseptal A

Diseptal B

Diseptal C

p-(p-Aminophenyl)-benzene-sulfonamide

Sulfanilanilide

1-Sulfanilamido-3, 5-dibromobenzene

1-Sulfanilamido-3, 5-dichloróbenzene

Sulfápyridine

2-(4'-Amino-3', 5'-dibromo-benzene-sulfonamido)-pyridine

2-Sulfanilamido-6-methyl-pyridine

3-Sulfanilamido-2, 6-lutidine

2-Sulfanilamido-5-chloro-pyridine

2-Sulfauilamido-5-bromo-pyridine

2-Sulfanilamido-5-amino-pytidine

Sulfathiazole

Sulfamethylthiazole

2-(4'-Amino-3', 5'-dibromobenzene-sulfonamido)-5-bromo-thiazole

4-Sulfanilamido-1-phenyl-2, 3-dimethyl-5-pyrazolone

2-Sulfanilamido-benzothiazole

N-Dimethyisulfanilamide

Marfanilamide

3-Carboxy-4-aminoazobenzene-4'-sulfonamide

Rubiazol

Proseptasine

2-( $\mathrm{N}^{4}$-Sulfanilylsulfanilamido $)-4-$ methylthiazole

$\mathrm{N}^{\prime}$-Dimethyl- $\mathrm{N}^{4}-\left(\mathrm{N}^{\prime 4}\right.$-sülfanilylsulfanil $)$-sulfanilamide

Phonocasil

2-Sulfanilamido-6-methoxy-benzothiazole

2-Sulfanilamido-6-ethoxy-benzothiazole

2-Sulfanilamido-5-bromo-benzothiazole

2-Sulfanilamido-6-choloro-benzothiazole

7-(4'-Sulfonamidophenylazo)-6-hydroxy-benzothiazole

7-(4'-Sulfonamidophenylazo)-2-methyl-6-hydroxy-benzothiazole

2-Sulfanilamido-pyridine

2-Sulfanilamido-4-methylpyrimidine

2-Sulf anilamido-4, 6-dimethyl-pyrimidine

Calco-acimidone

1-Sulfanilamido-4-bromo-naphthalene

1-Sulfanilamido-5, 8-dibromo-naphthalene

Table 16

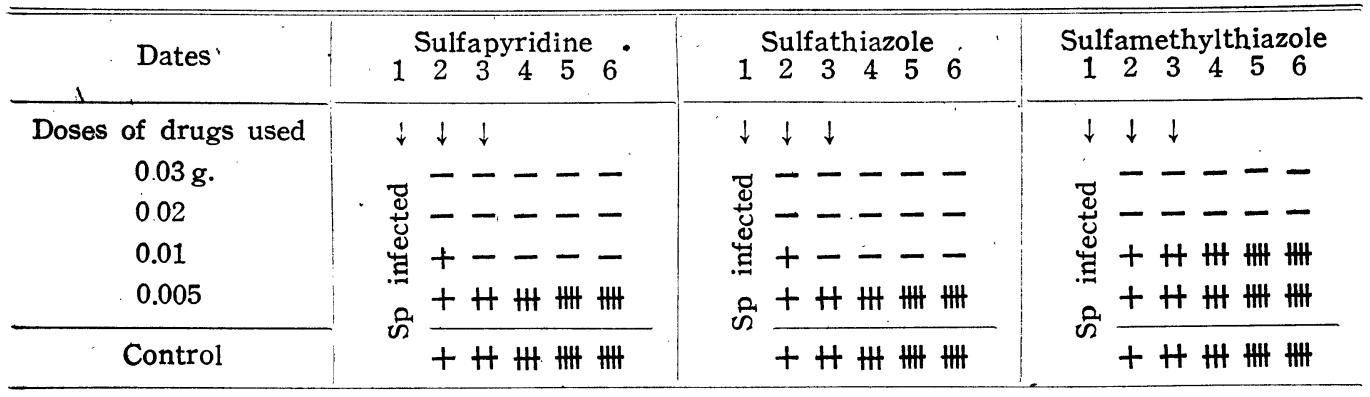


The activity of sulfapyridine is the same as that of sulfathiazole but sulfamethylthiazole has less action than these two drugs.

\section{(2) Diphenylsulfone and approximate compounds.}

Eight compounds were tested with negative results. (Table 17)

Table 17

4, 4'-Diamidino-stilbene

4, 4'-Diamidino-diphenylsulfone

4, 4'-Diamidino-diphenylsulfoxyde

4, 4'-Diguanidino-diphenylsulfone

4, 4'-Diguanidino-diphenylsulfoxyde

$4,4^{\prime}$-Diguanidino-diphenylsulfide

Promin

4-Aminophenyl-3'-aminopyridyl-(6')-sulfone-N, $\mathrm{N}^{\prime}$-didextrose sulfonate

\section{Chemotherapy of TRYpanosomiasis}

Experimental chemotherapy of trypanosomiasis (Trypanosoma gambiense) in white mice was carried out. It has been known that the effective drugs against trypanosomiasis of mice show trypanocidal action in human cases as well, and some effect in leishmaniasis. We examined three groups of chemical compounds in our investigation.

(a) Antimony compounds

New antimony compounds made by Dr. K. Tsuda were examined and compared with fouadin and neostibosan. (Table 18, 19) Nos. 104, 105, 106 and 110 seemed to be available for practical use.

Table 18

No. 101 8-Oxychinoline-5, 7-disulfonate sodium antimony compound

No. 102 8-Oxychinoline-5, 7-disulfonate diethylamine antimony compound

Do. 103 8-Oxychinoline-5-sulfonate-sodium antimony compound

No. 104 7-Iodo-8-oxychinoline-5-sulfonate sodium antimony compond

No. 105 7-Bromo-8-oxychinoline-5-sulfonate sodium antimony compound

No. 106 7-Chloro-8-oxychinoline-5-sulfonate sodium antimony compound

No. 109 7-Amino-8-oxychinoline-5-sulfonate sodium antimony compound

No. 110 7, 8-Dioxychinoline-5-sulfonate sodium antimony compond

Table 19

\begin{tabular}{|c|c|c|c|c|c|}
\hline Drugs & M.T.D. & M.E.D. & M.C.D. & $\begin{array}{l}\text { M.T.D. } \\
\text { M.E.D. }\end{array}$ & $\begin{array}{l}\text { M.T.D. } \\
\text { M.C.D. }\end{array}$ \\
\hline No. 101 & $1.5 \mathrm{mg}$. & $1.0 \mathrm{mg}$. & $1.5 \mathrm{mg}$. & $1: 1.5$ & $1: 1$ \\
\hline No. 102 & 1.0 & 1.0 & - & - & - \\
\hline No. 103 & 0.2 & 0.2 & - & $1: 1$ & - \\
\hline No. 104 & 0.8 & 0.13 & 0.13 & $1: 6.1$ & $1: 6.1$ \\
\hline No: 105 & 0.5 & 0.13 & 0.25 & $1: 3.8$ & $1: 2$ \\
\hline No. 106 & 0.6 & 0.15 & 0.25 & $1: 4$ & $1: 2.4$ \\
\hline No. 109 & 2.5 & 2.5 & - & $1: 1$ & - \\
\hline No. 110 & 0.9 & 0.35 & 0.4 & $1: 2.6$ & $1: 2.2$ \\
\hline Fouadin & 6.4 & 0.4 & 0.8 & $1: 1.6$ & $1: 8$ \\
\hline Neostibosan & 25.0 & 8.0 & 8.0 & $1: 3.1$ & $1: 3.1$ \\
\hline
\end{tabular}




\section{(b) Sulfonamide compounds}

Forty one sulfonamide compounds tested had no action on trypanosome infection. (Table 20)

Table 20

Sulfanilamide

Sulfanilylguanidine

p-Aminobenzolsulfonacetamide

Diseptal A

Diseptal B

Diseptal C

p-(p-Aminophenyl)-benzene-sulfonamide

Sulfanilanilide

1-(Sulfanilamido)-3, 5-dibromobenzene

1-(Sulfanilamido)-3, 5-dichlorobenzene

Sulfapyridine

2-Sulfanilamido-6-methylpyridine

3-Sulfanilamido-2, 6-lutidine

2-Sulfanilamido-5-chloropyridine

2-Sulfaniamido-5-bromopyridine

Sulfathiazole

Sulfanilamido-4-methyl-5-carbathoxy-thiazole

4-Sulfanilamido-1-phenyl-2, 3-dimethyl-5-pyrazolone

2-Sulfanilamido-benzothiazole

$\mathrm{N}$-Dimethylsulfanilamide

Marfanilamide

Prontosil rubrum

3-Carboxy-4-aminoazobenzene-4'-sulfonamide

Rubiazol

Proseptasine

2-(N-Sulfanilylsulfanilamido)-4-methyl-thiazole

$\mathrm{N}^{\prime}$-Dimethyl-N-(N'-sulfanilylsulfanil)-sulfanilamide

$2-\left(\mathrm{N}^{4}-\left(\mathrm{N}^{\prime 4}-\right.\right.$ Sulfanilyl-sulfanilyl)-șulfanilamido $)-4-$ methylthiazole

$\mathrm{N}^{\prime}$-Dimethyl- $\mathrm{N}^{4}-\left(\mathrm{N}^{\prime 4}-\left(\mathrm{N}^{\prime \prime 4}-\right.\right.$ sulfanilyl-sulfanilyl)-sulfanilyl)-sulfanilamide

Phonocasil

2eSulfanilamido-6-methoxy-benzothiazole

2-Sulfanilamido-6-ethoxy-benzothiazole

2-Sulfanilamido-5-bromo-benzothiazole

2-Sulfanilamido-6-chloro-benzothiazole

7-(4'-Súlfonamidophenylazo)-6-hydroxy-benzothiazole

7-(4'-Sulfonamidophenylazo)-2-methyl-6-hydroxy-benzothiazole

2-Sulfanilamido-pyrimidine

2-Sulfanilamido-4-methylpyrimidine

2-Sulfanilamido-4, 6-dimethylpyrimidine

Calco-acimidon

\section{(c) Diphenylsulfone and approximate compounds}

Several new compounds, submitted by Dr. S. Sugasawa, were examined. The effect of diamidino-stilbene against Trypanosoma rhodesiense had already been observed by Yorke (1940). We tested the activity of diamidino-stilbene on Trypanosoma gambiense with the same results as those which he had observed.

There are still newer compounds which were found to have trypanocidal action according to our experiments. (Table 21, 22) 
Table 21

D 1<smiles>NC1CCC(OS(=O)(=O)C2CCC(N)CC2)CC1</smiles>

non-effective

D. 2<smiles>NC1CCC(S(=O)(=O)C2CCC(N)CC2)CC1</smiles>

D 3

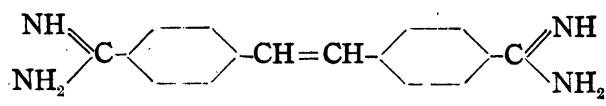

effective

D 4<smiles>N=C(N)C1CCCC(CCC2CCC(C(=N)N)CC2)CC1</smiles>

little effective

D 5<smiles>N=C(N)C1CCCC(=[Se]2CCC(C(=N)N)CC2)CC1</smiles>

non-effective

D 6<smiles>N=C(N)C1CCCC(S(=N)(=N)C2CCC(C(=N)N)CC2)CC1</smiles>

D 7

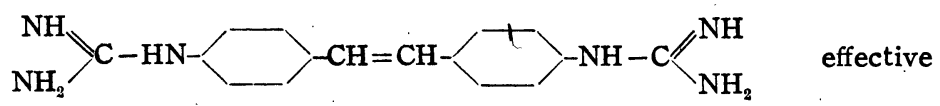

D 8

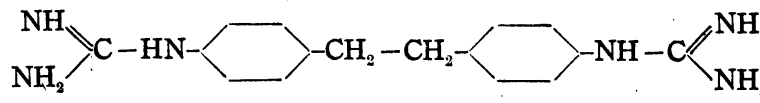

D 9

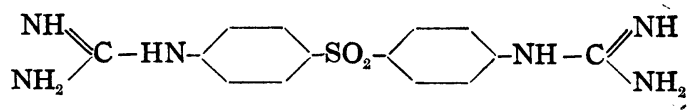

D10

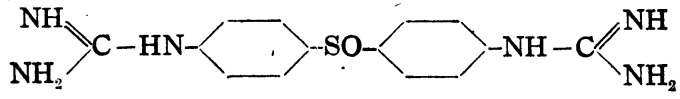

D11

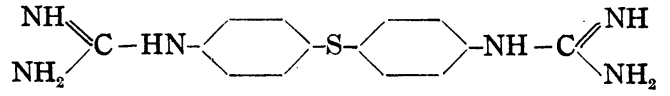

(These drugs are shown in table 8)

Table. 22

\begin{tabular}{c|c|c|c|c|c}
\hline \hline Drugs & M.L.D. & M.T.D. & M.E. & M.C.D. & M.T.D. \\
\cline { 1 - 4 } D 1 & - & - & - & - & M.C.D. \\
\hline D 2 & - & - & - & - & - \\
D 3 & $1.5 \mathrm{mg}$ & $13 \mathrm{mg}$ & $0004 \mathrm{mg}$ & $0.05 \mathrm{mg}$ & $1: 26$ \\
D 4 & 09 & 0.8 & 07 & - & - \\
D 5 & 2.5 & 2.0 & - & - & - \\
D 6 & 1.5 & 1.0 & - & - & - \\
D 7 & 1.2 & 1.0 & 0.5 & $?$ & - \\
D 8 & 06 & 0.5 & 0.2 & $:$ & $?$ \\
D 9 & 09 & 0.8 & 03 & 0.6 & $1: 1.3$ \\
D 10 & 18 & 1.5 & 0.2 & 0.3 & $1: 5$ \\
D 11 & 04 & 0.3 & 005 & 0.3 & $1: 1$ \\
\hline
\end{tabular}




\section{CHEMOTHERAPY OF LEISHMANIASIS}

\section{(1) Diphenylsulfone and approximate compounds.}

From this work we concluded that 4,4'-diguanidino-diphenylsulfoxyde is the most readily availble drug for trypanosomiasis.

Accordingly, we applied this new drug in human leishmaniasis, since the drug which has trypanocidal activity shows also some action against kala-azar. In one course a total dose $1.28 \mathrm{~g}$. of $4,4^{\prime}$-diguanidino-diphenylsulfoxyde was given intravenously into nine human kala-azar cases for 3 weeks on every other day; eight out of these cases demonstrated fairly good results. Thus a currative result will be obtained by repeating the administration of this drug, but leucopenia seems to be an attendant conflication. Therefore we are endeavoring to change the nature of this drug.

\section{(2) Sulfonamide compounds.}

Ten sulfonamide compounds were tested in kala-azar infection of striped squirrel, but no effect was found.

\section{(3) Antimony compounds.}

Nos. 104, 110 were tested in squirrel kala-azar with no effect but neostibosan was tried as a control with complete cure of the infection. This means the pentavalent antimony is more curative than the trivalent compounds.

\section{CHEMOTHERAPY OF ENTAMOEBIASIS}

For the purpose of examining drugs we used cultivated Entamoeba histolytica in vitro. If the drug prevents the growth of amoebae, we suppose it to be an effective drug against amoebiasis. Through our investigations the well-known drugs, emetine hydrochloride and yatren, were used for control.

\section{(1) Quinoline derivatives.}

Yatren and other compounds were tested, with a result that Nos. 104, 105, 106 were three times as effective as yatren. (Table 23, 24)

Entamoeba histolytica was cultivated in Dobell-Laidlaw's medium, the fluid containing: drugs in several concentrations. The drugs used are as follows:

Yatren 7-Jodo-8-oxyquinoline-5-sulfonate

No. 104 7-Jodo-8-oxyquinoline-5-sulfonate sodium antimony compound

No. 105 7-Bromo-8-oxyquinoline-5-sulfonate sodium antimony compound

No. 106 7-Chloro-8-oxyquinoline-5-sulfonate sodium antimony compound

No. 110 7, 8-Dioxyquinoline-5-sulfonate sodium antimony compound

Table 23 Growth of E. histolytica

\begin{tabular}{c|c|c|c|c|c}
\hline Drug & Concentration & $24 \mathrm{hrs}$ & $48 \mathrm{hrs}$ & $72 \mathrm{hrs}$ & resu ts \\
\cline { 2 - 6 } Yatren & $1: 1.000$ & - & - & - & effective \\
& $1: 2.000$ & + & - & - & $"$ \\
& $1: 3.000$ & $H$ & + & + & non-effective \\
& $1: 10.000$ & $\mathrm{H}$ & $\mathrm{H}$ & + & $"$ \\
\hline
\end{tabular}




\begin{tabular}{|c|c|c|c|c|c|}
\hline Drug & Concentration & $24 \mathrm{hrs}$ & $48 \mathrm{hrs}$ & $72 \mathrm{hrs}$ & results \\
\hline No. 104 & $\begin{array}{l}1: 3.000 \\
1: 5000 \\
1: 7000 \\
1: 10.000\end{array}$ & $\begin{array}{l}- \\
- \\
+ \\
+\end{array}$ & $\begin{array}{l}- \\
- \\
\pm \\
+\end{array}$ & $\begin{array}{l}- \\
- \\
-\end{array}$ & $\begin{array}{c}\text { effective } \\
\prime \prime \\
" \prime \\
\text { non-effective }\end{array}$ \\
\hline No. 105 & $\begin{array}{l}1: 3000 \\
1: 5000 \\
1: 7000 \\
1: 10.000\end{array}$ & $\begin{array}{l}- \\
- \\
+ \\
+\end{array}$ & $\begin{array}{l}- \\
- \\
+\end{array}$ & $\begin{array}{l}- \\
- \\
- \\
+\end{array}$ & $\begin{array}{c}\text { effective } \\
\prime \prime \\
\prime \prime \\
\text { non-effective }\end{array}$ \\
\hline No. 106 & $\begin{array}{l}1: 3.000- \\
1: 5000 \\
1: 7000 \\
1: 10000\end{array}$ & $\begin{array}{l}- \\
- \\
+ \\
+\end{array}$ & $\begin{array}{l}- \\
- \\
\pm \\
+\end{array}$ & $\begin{array}{l}- \\
- \\
+\end{array}$ & $\begin{array}{c}\text { effective } \\
" \\
" \\
\text { non-effective }\end{array}$ \\
\hline $\begin{array}{l}\text { No. } 110 \\
\text { Control }\end{array}$ & $\begin{array}{l}1: 1.000 \\
1: 3.000\end{array}$ & $\begin{array}{l}+ \\
H \\
H\end{array}$ & $\begin{array}{l}+ \\
H \\
H\end{array}$ & $\begin{array}{l}+ \\
+ \\
\text { H }\end{array}$ & " \\
\hline
\end{tabular}

Table 24

\begin{tabular}{|c|c|c|c|c|c|c|c|c|}
\hline . & $1: 1000$ & $1: 2000$ & $1: 3000$ & $1: 5000$ & $1: 7000$ & $1: 10000$ & $1: 50000$ & $1: 100000$ \\
\hline $\begin{array}{l}\text { Emetine } \\
\text { Osvarsan } \\
\text { Yatren }\end{array}$ & $\begin{array}{l}0 \\
0\end{array}$ & $\begin{array}{l}0 \\
0\end{array}$ & $\begin{array}{l}\Delta \\
\Delta\end{array}$ & & & 0 & $\Delta$ & $x$ \\
\hline $\begin{array}{l}\text { No. } 104 \\
\text { No. } 105 \\
\text { No. } 106 \\
\text { No. } 110\end{array}$ & $\Delta$ & & $\begin{array}{l}0 \\
0 \\
0 \\
x\end{array}$ & $\begin{array}{l}0 \\
0 \\
0\end{array}$ & $\begin{array}{l}0 \\
0 \\
0\end{array}$ & $\begin{array}{l}\Delta \\
\Delta \\
\Delta\end{array}$ & & \\
\hline
\end{tabular}

(2) Sulfonamide compounds

Thirty one sulfonamide compounds were tested; 2 of these seemed to be practically effective for amoebiasis. (marked $\mathrm{O}$ in Table 25, 26)

Table 25

Sulfanilamide

Sulfanilylguanidine

Diseptal A

Diseptal B

Diseptal C

$\mathrm{p}-(\mathrm{p}$-Aminophenyl)-benzene-sulfonamide

Sulfanilanilide

1-Sulfanilamido-3, 5-dichlorobenzene

Sulfapyridine

2-Sulfanilamido-5-chloropyridine

Sulfathiazole

2, 3-Dimethyl-5-pyrazolone?

1-Sulfanilamido-3, 5-dibromobenzene

3-Sulfanilamido-quinoline 
2-Sulfanilamido-benzothiazole

N-Dimethylsulfanilamide

Marfanilamide

3-Carboxy-4-aminoazobenzene-4'-sulfonamide

Rubiazol

Proceptasine

$\mathrm{N}^{\prime}$-Dimethyl- $\mathrm{N}^{4}-\left(\mathrm{N}^{4}-\right.$ sulfanilylsulfanil $)$-sulfanilamide

$\mathrm{N}^{\prime}$-Dimethyl- $\mathrm{N}^{4}-\left(\mathrm{N}^{\prime 4}-\left(\mathrm{N}^{/ / 4}-\right.\right.$ sulfanilylsulfanilyl)-sulfanilyl)-sulfanilamide

Phonocas'1

2-Sulfanilamido-pyrimidine

3, 5-Dibromo-sulfanilamide

2-Sulfanilamido-5-amino-pyridine

Calcoacimidon

p-Aminobenzol-sulfonacetamide

2-Sulfonamido-5-bromo-benzothiazole

1-Sulfanilamido-4-bromo-naphthalene

1-Sulfanilamido-5,8-dibromo-naphthalene

Table 26

\begin{tabular}{|c|c|c|c|c|c|c|c|c|c|c|}
\hline 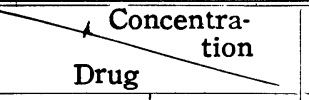 & $1: 200$ & $1: 300$ & $1: 500$ & $1: 1,000$ & $1: 2,000$ & $1: 3,000$ & $1: 5,000$ & $1: 10,000$ & $1: 50,000$ & $1: 100,000$ \\
\hline Sulfanilamide & 0 & $\Delta$ & $\times$ & & & & & & & \\
\hline $\begin{array}{l}\text { 1-Sulfanilamido- } \\
\text { 3,5-dibromobenzene }\end{array}$ & .0 & $\Delta$ & $x$ & & & & & & & \\
\hline $\begin{array}{l}\text { 1-Sulfanilamido- } \\
\text { 3,5-dichlorobenzene }\end{array}$ & 0 & 0 & 0 & 0 & $x$ & $x$ & $x$ & $x$ & , & \\
\hline $\begin{array}{l}\text { 3-Carboxy-4-amino } \\
\text { azobenzene-4/ } \\
\text { sulfonamide }\end{array}$ & 0 & 0 & 0 & $\Delta$ & $x$ & $x$ & $x$ & $x$ & & \\
\hline Phonocasil . & O & 0 & $\triangle$ & & & & & & & \\
\hline $\begin{array}{l}\text { 2-Sulfonamido-5- } \\
\text { bromo-benzothiazole }\end{array}$ & 0 & $\Delta$ & $x$ & & & & & & & \\
\hline Proceptasine & 0 & $\triangle$ & $x$ & & & & & & & \\
\hline Yatren & & & & $\mathrm{O}$ & O & $\Delta$ & . & & & \\
\hline $\begin{array}{l}\text { Emetine } \\
\text { hydrochloride }\end{array}$ & & & & & & & & 0 & $\Delta$ & $x$ \\
\hline
\end{tabular}

O Effective

$\Delta$ Slightly effective

$\times$ Non-effective

(3) Miscellaneous compounds

Nine miscellaneous compounds were tested resulting non-effective.

Phenothiazine

4, 4'-Diamidino-stilbene

4, 4'-Diamidino-diphenylsulfone

4, 4'-Diamidino-diphenylsulfoxyde

4, 4'-Diguanidino-diphenylsulfone

4, 4'-Diguanidino-diphenylsulfoxyde

4, 4'-Diguanidino-diphenylsulfide

Promin

4-Aminophenyl-3'-aminopyridyl-(6')-sulfone-N, $\mathrm{N}^{\prime}$-didextrose sulfonate 


\section{CHEMOTHERAPY OF TRICHOMONIASIS}

Trichomonas hominis, cultivated in Dobell-Laidlaw's medium, was used for testing effectiveness of the drugs. The drugs were diluted into the liquid part of the media, in which $T$. hominis was cultivated.

\section{(1) Quinoline derivatives}

Five quinoline derivatives were used, emetine hydrochlide and osvarsan were used for comparison. Nos. 104, 105, 106 were ten times as effective as yatren and little more than emetine hydrochloride. Therefore these three seem to be practically useful against Trichomoniasis. hominis. (Table 27)

Yatren 7-Jodo-8-oxyquinoline-5-sulfonate

No. 104 7-Jodo-8-oxyquinoline-5-sulfonate sodium antimony compound.

No. 105 7-Bromo-8-oxyquinoline-5-sulfonate sodium antimony compound

No. 106 7-Chloro-8-oxyquinoline-5-sulfonate sodium antimony componnd

No. 110 7,8-Dioxyquinoline-5-sulfonate sodium antimony compound

Table 27

\begin{tabular}{|c|c|c|c|c|c|c|c|}
\hline & $1: 1,000$ & $1: 2,000$ & $1: 3,000$ & $1: 5,000$ & $1: 7,000$ & $1: 10,000$ & $1: 20,000$ \\
\hline $\begin{array}{l}\text { Emetine } \\
\text { Osvarsan } \\
\text { Yatren }\end{array}$ & $\begin{array}{l}0 \\
0\end{array}$ & $\begin{array}{l}\times \\
\times\end{array}$ & & . & & $\Delta$ & $x$ \\
\hline No. 104 & 0 & 0 & 0 & 0 & 0 & 0 & $\times$ \\
\hline No. 105 & 0 & 0 & 0 & 0 & 0 & 0 & $\times$ \\
\hline No. 106 & 0 & 0 & 0 & 0 & 0 & 0 & $x$ \\
\hline No. 110 & 0 & 0 & 0 & $\Delta$ & $x$ & & \\
\hline
\end{tabular}

Table 28 Sulfonamide

Sulfanilamide

Sulfanilylguanidine

Diseptal A

Diseptal B

Diseptal C

p-(p-Aminophenyl)-benzene-sulfonamide

Sulfadibromobenzene

Sulfadichlorobenzene

Sulfapyridine

Sulfachloropyridine

Sulfathiazole

Sulfamethylthiazole

4-Sulfanilamido-1-phenyl-2, 3-dimethyl-5-pyrazolone

2-Sulfanilamido-quinoline

2-Sulfanilamido-benzothiazole

$\mathrm{N}^{4}$-Dimethylsulfanilamide

Marfanilamide

Prontosil rubrum

2,4-Oxyazobenzene-4'-Sulfonamide

3-Carboxy-4-aminoazobenzene-4'-sulfonamide

Rubiazole

Proseptasine 


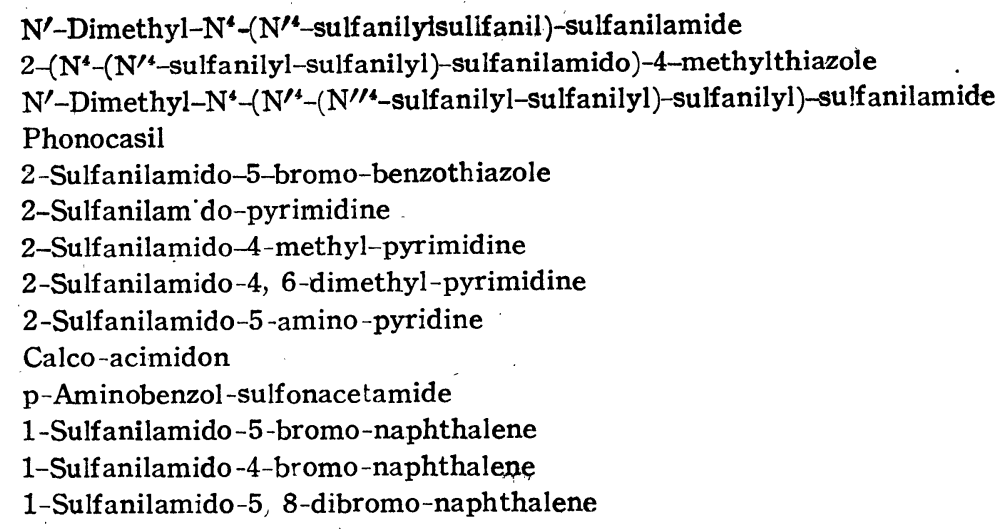

\section{(2) Sulfonamide compounds}

Thirty six sulfonamide compounds were tested, but none of them seemed to be of valve for trichomoniasis. (Table 28)

\section{(3) Diphenylsulfone and approximate compounds}

Eleven drugs were tested against Trichomonas hominis in culture. D3 and D7 w'ere found to be more potent than others, these two stilbene compounds, appearing to be effective against Trichomonas. Stilbene guanidine is more effective than stilbene amidine. (Table 29)

\section{Summary}

The researches on the chemotherapy of protozoal infections, malaria, spirochetosis, trypanosomiasis, leishmaniasis, entamebiasis and trichomoniasis, were carried out in this laboratory.

1. Oút of 53 sulfonamide compounds, sulfadibromobenzene and sulfadichlorobenzene were found effective on canary malaria; sulfadibromobenzene was applied on humạ tertian malaria with desirable results. Eight quinine derivatives, out of 42 tested, exhibited a malaricidal action on canary malaria, and 6-amino-dihydro-cinconidine showed satisfactory results in human tertian cases.

Dr. Asano's atebrin was found to be as effective as other types of atebrin on canary malaria and human tertian cases.

Chemical compoune of quininebisulfate and sulfadibromobenzene were also exami ned with effective result in both canary and human malaria.

Cepharanthin was found to be a provocative drug which can be applied for the purpose of complete cure of malaria when accompanied by malaricidal drugs.

2. Out of 43 sulfonamide compounds examined, sulfapyridine, sulfathiazole and sulfamethylthiazole were found to be effective in mice spirochetosis recurrentis.

7-Jado-8-oxyquinoline-5-sulfonate sodium antimony compound

7-Bromo-8-oxyquinoline-5-sulfonate sodium antimony compound

7-Chloro-8-oxyquinoline-5-sulfonate sodium antimony compound

T, 8-Dioxyquinoline-5-sulfonate sodium antimony compound showed satisfactory results in mice trypanosomiasis, 
Table 29

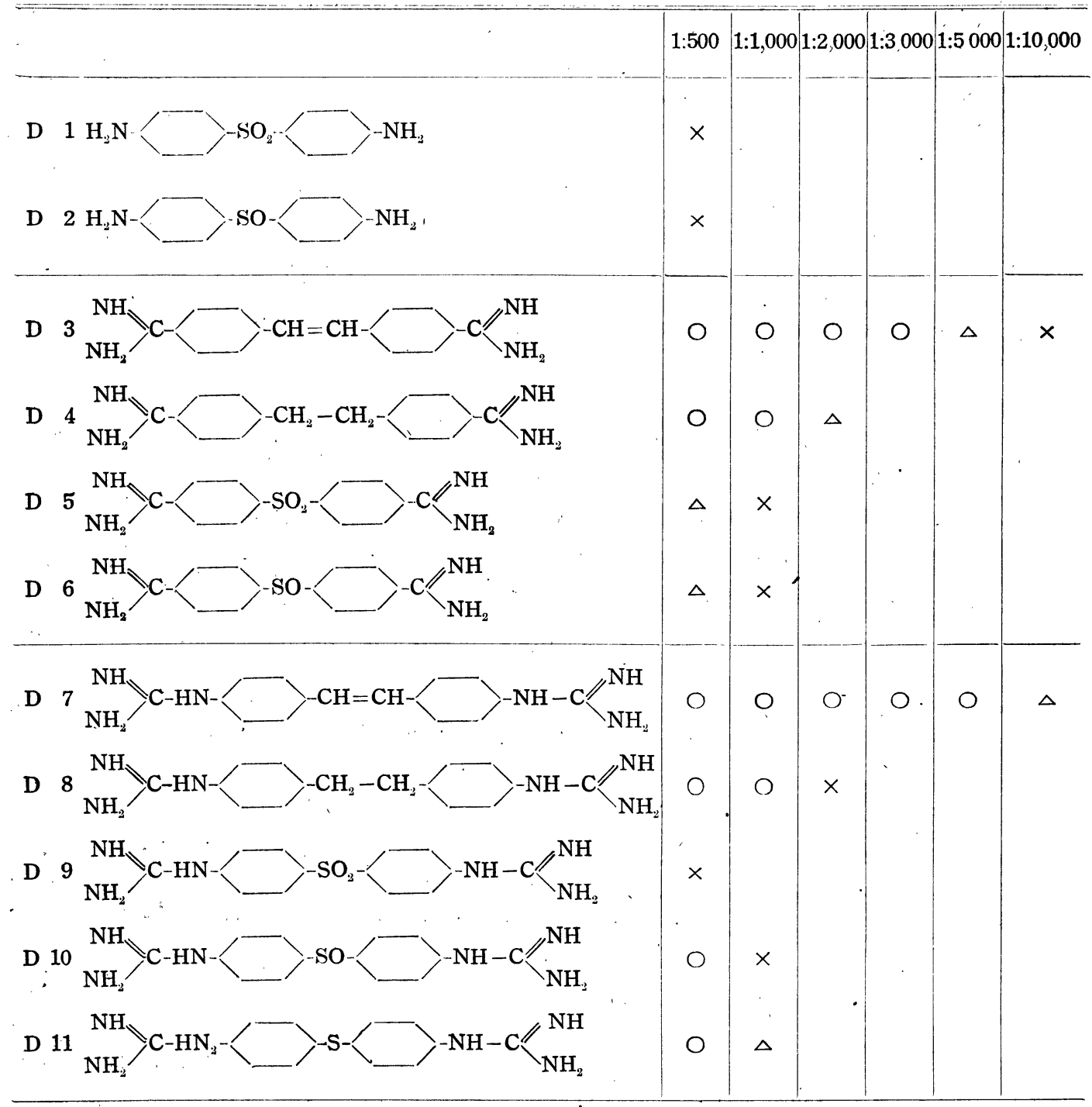

4. Diguanidino-diphenylsulfoxyde was applied in human kala-azar cases with good effects.

5. 7-Jodo-8-oxyquinoline-5-sulfonate sodium antimony compound, 7-kromo-8-oxyquinoline-5-sulfonate sodium antimony compound and 7-chloro-8-oxyquinoline-5sullonate sodium antimony compònd were found effective for entamebiasis.'

6. Three quinoline derivatives, 7-jodo-8-oxyquinoline-5-fulfonate sodium antimony compound, 7-bromo-8-oxyquinoline-5-sulfonate sodium antimony compound and 7chloro-8-oxyquinoline-7-sulfonate sodium antimony compound were found effective in trichomoniasis. Diamidino-stilbene and diguanidino-stilbene also showed satisfactry results in trichomoniasis. 
References

Ishii, N., Shimizu, S. and Tsuda, K.: The effects of sulfapyridine on experimental spirochaetosis recurrentis (in Japanese) Tokyo Iji Shinshi, No. 3143, pp. 1893-1896, 1939. Do. (in English) Jap. J. Exper. Med. Vol. 19, No. 1, 2, 3, pp. 5-9, 1941.

Ishii, N.: Studies on malaria II. Effects of sulfonamide compounds against malaria. (in Japanese) Jikken Igaku Zasshi, Vol. 25, No. 9, pp. 1130-1134. Do (in English) Jap. J. Exper. Med, Vol. 19, No. 5 pp. $151-155,1941$.

Ishii, N. and Yajima, Y.: Studies on malaria III. Effects of benzothiazole, benzothiazolone quinazolone derivatives against malaria. (in Japanese) Jikken Igaku Zasshi, Vol. 26, No. 11, pp 918-921, 1942.

Ishii, N, and Kimura, K.: The effects of sulfonamide compounds on experimental spirochaetosis recurrentis. (in Japanese) Jikken Igaku Zasshi, Vol. 26, No. 4, pp. 1-3, 1942.

Ishii, N.: 'Experimental studies on chemotherapy of protozoal infections. (in Japanese) Nippon Igaku, No. 3395 pp. 977-985; No. 3396, pp. 999-1003, 1944.

Yorke W.: Recent work on the chemotherapy of protozoal infections. Royal Soc. Trop. Med. and Hyg., Vol. 33, No. 5, 1940

\title{
STUDIES ON THE MECHANISM OF THE AGGLUTINATION I. ON THE LATTICE THEORY
}

\author{
Hamao UMEZAWA \\ National Institute of Health of Japan, Tokyo
}

The field of the immunology is so extensive and the mechanism of the immunological reactions is so complex that no one has found it possible to induce an inclusive theory.

It seems to be too adventurous to explain the mechanism of the infection and prevention now with the aid of the chemistry and physics. But in the field of the simplest reactions, precipitation and agglutination, the recent informations of the chemistry seem to be available and the mechanism of these simple reactions will be useful for the study of the more complex one, as the most, important phenomenon, the specific combination, is common to all reactions.

At about the time when I have undertaken this work (1939), a splendia theory was advanced by Pouling (1) (1940).

Almost all our experiments were performed just before the war, but could not be published owing to the difficult communication in the recent several years. Our results seem to almost conform to Pauling's theory, and contribute to the complex immunological reactions.

In tris report an experiment to test Marrack's Hypothesis (2) (Lattice theory) is described. Marrack's Lattice theory has been confirmed by several workers, Heidelberger (3), Topley, Wilson, and Duncan (4), but challenged by Abramson (5), Hooker and Boyd (6), and Haurowitz (7).

Though the experiment by Topley and others is very simple the result has a great meaning in deciding whether there are two stages, specific combination and non-specific aggregation, or only one specific stage in the course of agglutination. 